\author{
Linlin ZHANG, Post graduate \\ Lecturer Yizhuo LI, (First Corresponding Author) \\ E-mail: yizhuo_li@qq.com \\ Associate Professor Xu WU, PhD (Second Corresponding Author) \\ E-mail: wuxuphd@foxmail.com \\ Lecturer Qianying Feng \\ School of Business \\ Chengdu University of Technology
}

\title{
RESEARCH ON FRACTAL PORTFOLIO MODEL UNDER POWER-LAW DISTRIBUTION OF RETURN RATE
}

\begin{abstract}
Based on the fact that the tail of the security yield follows a power-law distribution, this paper first builds on the fractal dimension calculation idea to construct two fractal statistical measures: fractal expectation and fractal variance. Then, using fractal expectation and fractal variance as the measurement indicators of returns and risks, a portfolio model under fractal was constructed, and the analytical solution of the model was given. Finally, through empirical research, it is found that fractal expectation and fractal variance can overcome the defect that the traditional mean and traditional variance cannot be applied to the power law distribution of the tail of the return rate, is conducive to improving the performance of the investment portfolio.

Keywords: fractal expectation, fractal variance, power-law distribution, fractal portfolio model.
\end{abstract}

\section{JEL Classification: G11}

\section{Introduction}

As is known to all, finance is the core of the modern economic system, and the efficient and orderly operation of the financial system is crucial to economic development, and the size, trend and volatility of financial markets (Peretz 1971; Ankargren et al. 2017). Traditional financial theory based on the efficient market hypothesis believes that the elements of the financial system show a simple linear relationship, but in fact the real financial system is a complex nonlinear one. The rate of return on securities does not present normal distribution under the efficient market hypothesis, but often exhibits "thick tail" characteristics (Scheinkman 1994; Wang et al. 2011). Some studies have shown that the "thick-tailed" nature of returns obey the power-law distribution. At this time, if traditional means and variances are still used as measures of risk and return, and financial decisions are

DOI: 10.24818/18423264/55.1.21.14 
studied accordingly, it will inevitably affect the accuracy of the research conclusion (Cont 2001; Yuan et al. 2009; Lux et al. 2016). Therefore, under the fact that the tail of the rate of return obeys the power-law distribution, it is necessary to reconstruct the recognition measures of investment return and risk for the characteristics of the power-law distribution for making financial decisions.

As the core of financial decision-making theory, investment portfolio plays a pivotal role. Related researches have not only promoted the development of modern finance, but also is one of the boosters of modern financial theory research. In this sense, to study financial decision-making based on power-law distribution, it is necessary to study investment portfolio under such circumstance. For investors, an effective investment portfolio can maximize the return under a given risk, or minimize the risk under a given return (Samuelson 1967; Markowitz 1991). As early as in 1952, Markowitz incorporated both mean and variance into the portfolio research framework, pioneered the construction of a mean-variance portfolio model, and laid the foundation for modern portfolio theory (MPT) (Markowitz 1952). Subsequently, a large number of scholars carried out effective research based on this model (Li et al. 2013; Jung et al. 2015; Ortobelli et al. 2017; Hany 2019). They not only promoted the improvement of MPT and the development of modern financial theory, but also opened up a new chapter of investment portfolio research, marking a beginning of modern financial theory. As mentioned earlier, the core issue of portfolio research is to distribute wealth among different assets in order to diversify risks and ensure returns. Therefore, accurate measurement of risks and returns is a prerequisite for building an effective portfolio model. In addition, some scholars have pointed out that a tiny miss in the measurement of risk and return will definitely lead to poor performance of portfolio management (Black 1991). Hence, building a portfolio model requires accurate measurement of risks and returns.

However, the real financial system is essentially a complex hybrid one, which incubates non-linear markets. Therefore, related researches conducted under the assumption that security prices are linearly and normally distributed, is no longer persuasive (Brandtner et al. 2013; Goh et al. 2012; Zhao et al. 2016; Alexander et al. 2017). And a large number of studies have shown that the distribution of security prices has a "thick tail" feature, and the tail actually follows a power-law distribution. At this time, the distribution based on the efficient market hypothesis may present no or indefinite measurement which can cause a flaw on accurately calculating risks and returns (Lim et al. 2007; Wang et al. 2009; Zhang et al. 2018). What is more, since the real financial system is a complex hybrid one, it is urgent to incorporate the real distribution of security prices in the financial market, that is, the power-law distribution, into the research framework. In other words, under the realistic background that security prices show fractal characteristics, only by using the fractal statistical analysis method to build an investment portfolio can we accurately measure the returns and risks of security prices (Samorodnitsky et al. 1996; Wu et al. 2015). 
Based on this, in order to accurately measure the returns and fractal portfolio model risks of security prices, this article starts from the fact that markets are non-linear when the actual financial system is a complex hybrid one, security prices and the tail of the securities yield rate follows the power law distribution, and constructs a portfolio model with fractal expectation and fractal variance (fractal portfolio model) basing on fractal ideas. The related operation rules are explored and the analytical solution of the model is given. In the empirical process, after comparing performance with Markowitz Mean-Variance Model, it is found that the performance under the fractal portfolio model is better than that under the traditional Markowitz Mean-Variance Model, which shows that the fractal portfolio model is more effective.

\section{Fractal statistical measure and construction of fractal portfolio model}

\subsection{Construction of fractal statistical measures}

$\rho(x)$ can be defined as the density function of the random variable $X . x_{0}$ , $\rho_{0}$ and $\alpha$ are constants greater than zero. When $x \geq x_{0}$, the $\rho(x)=\rho_{0} x^{-\alpha}$ holds; when $x<x_{0}, \rho(x)=0$, the expectation $E(X)=\int_{-\infty}^{\infty} x \rho(x) d x$ and variance $\operatorname{Var}(X)=E\left(X^{2}\right)-[E(X)]^{2}$ of the random variable $X$ may not exist. As a result, asset returns and risks cannot be measured using traditional expectations or traditional variances. In order to accurately measure the returns and risks of security prices, the method used by fractal theory to handle the length of the curve (Mandelbrot 1967; Wu et al. 2015) can be referred to in order to construct a measure of returns and risks, that is, the fractal expectation and fractal variance. Specifically, the array $\left\langle E_{X}, e_{X}\right\rangle$ can be defined by Eq.(1) to reflect the fractal expectations, which can be written as $E_{f}(X)=\left\langle E_{X}, e_{X}\right\rangle$. Similarly, the fractal variance can be defined by the array $\left\langle V_{X}, v_{X}\right\rangle$ defined by Eq.(2), so the fractal variance can be recorded as $\operatorname{Var}_{f}(X)=\left\langle V_{X}, v_{X}\right\rangle$.

$$
\begin{aligned}
\left\langle E_{X}, e_{X}\right\rangle & =\left\langle(2-\alpha)^{-1} \rho_{0}, 2-\alpha\right\rangle \\
\left\langle V_{X}, v_{X}\right\rangle & =\left\{\begin{array}{l}
\left\langle(3-\alpha)^{-1} \rho_{0}, 3-\alpha\right\rangle, \alpha>1 \\
\left\langle 2^{-1} \rho_{0}-\rho_{0}^{2}, 2\right\rangle, \alpha=1 \\
\left\langle(2-\alpha)^{-2} \rho_{0}^{2}, 4-2 \alpha\right\rangle, \alpha<1
\end{array}\right.
\end{aligned}
$$

According to the above definition of the fractal statistical measure, let $a$ and $b$ be arbitrary constant. It is easy to prove that the fractal expectations $E_{f}(X)$ and $E_{f}(Y)$ of the random variables $\operatorname{Var}_{f}(X)$ and $\operatorname{Var}_{f}(Y)$, in keeping with the 
fractal variances $\operatorname{Var}_{f}(X)$ and $\operatorname{Var}_{f}(Y)$ are for any $q \in R$, the calculation rule shown as Eq.(3) and Eq.(4) hold.

$$
\begin{aligned}
& \left\{\begin{array}{l}
E_{f}(X)<E_{f}(Y) \Leftrightarrow\left(e_{X}<e_{Y}\right) \cup\left[\left(e_{X}=e_{Y}\right) \cap\left(E_{X}<E_{Y}\right)\right] \\
E_{f}(X) E_{f}(Y)=\left\langle E_{X} E_{Y}, e_{X}+e_{Y}\right\rangle \\
E_{f}(a X+b Y)=a E_{f}(X)+b E_{f}(Y)=\left\{\begin{array}{l}
\left\langle b E_{Y}, e_{Y}\right\rangle, e_{X}<e_{Y} \\
\left\langle a E_{X}, e_{X}\right\rangle, e_{X}>e_{Y} \\
\left\langle a E_{X}+b E_{Y}\right\rangle, e_{X}=e_{Y}
\end{array}\right. \\
E_{f}(X)\left[E_{f}(Y)\right]^{q}=\left\langle E_{X} E_{Y}^{q}, e_{X}+q e_{Y}\right\rangle
\end{array}\right. \\
& \int \operatorname{Var}_{f}(X)<\operatorname{Var}_{f}(Y) \Leftrightarrow\left(v_{X}<v_{Y}\right) \bigcup\left[\left(v_{X}=v_{Y}\right) \cap\left(V_{X}<V_{Y}\right)\right] \\
& \operatorname{Var}_{f}(X) \operatorname{Var}_{f}(Y)=\left\langle V_{X} V_{Y}, v_{X}+v_{Y}\right\rangle
\end{aligned}
$$

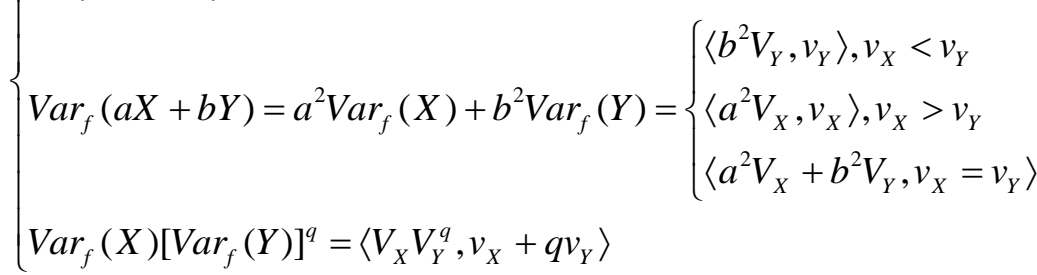

It can be seen that the fractal dimension idea can be used to construct the fractal expectation and fractal variance, based on the fact that the security yield follows the power-law distribution under the realistic background that markets are non-linear when the real financial system is a complex hybrid one. And by incorporating the fractal expectation and fractal variance into the mean-variance criterion, a portfolio model under fractal can be constructed to realize the dispersion of risk and increase of returns under the power-law distribution of security returns.

\subsection{Construction of fractal portfolio model}

It can be seen from the foregoing that the optimal investment portfolio is the one that maximizes the utility of investors by allocating investment weights to different assets (Markowitz 1952). Specifically, if $M 、 N$ are used as the two risk assets to construct the risk asset portfolio $P$, and investment funds are allocated according to the weights $\omega_{M} 、 \omega_{N}=1-\omega_{M}$ respectively, then the Eq.(5) is used to maximize the slope $S_{P}$ of the capital allocation line of the venture portfolio $P$. In this way, the optimal risk asset portfolio can be constructed in the traditional sense. Among them, $F$ is a risk-free asset; $r_{M} 、 r_{N} 、 r_{F}$ represent the yields of $M 、 N$ 、 $F$ respectively, written as $R_{M}=E\left(r_{M}\right)-E\left(r_{F}\right)$ and $R_{N}=E\left(r_{N}\right)-E\left(r_{F}\right)$. Consequently, the weight of the investment $\omega_{M} 、 \omega_{N}$ can be solved by Eq.(6) as: 


$$
\begin{aligned}
& \max S_{P}=\frac{\omega_{M} E\left(r_{M}\right)+\omega_{N} E\left(r_{N}\right)-E\left(r_{F}\right)}{\omega_{M}^{2} \operatorname{Var}\left(r_{M}\right)+\omega_{N}^{2} \operatorname{Var}\left(r_{N}\right)+2 \omega_{M} \omega_{N} \operatorname{Cov}\left(r_{M}, r_{N}\right)} \\
& \left\{\begin{array}{l}
\omega_{M}=\frac{R_{M} \operatorname{Var}\left(r_{N}\right)-R_{N} \operatorname{Cov}\left(r_{M}, r_{N}\right)}{R_{M} \operatorname{Var}\left(r_{N}\right)+R_{N} \operatorname{Var}\left(r_{M}\right)-\left(R_{M}+R_{N}\right) \operatorname{Cov}\left(r_{M}, r_{N}\right)} \\
\omega_{N}=\frac{R_{N} \operatorname{Var}\left(r_{M}\right)-R_{M} \operatorname{Cov}\left(r_{M}, r_{N}\right)}{R_{M} \operatorname{Var}\left(r_{N}\right)+R_{N} \operatorname{Var}\left(r_{M}\right)-\left(R_{M}+R_{N}\right) \operatorname{Cov}\left(r_{M}, r_{N}\right)}
\end{array}\right.
\end{aligned}
$$

Similarly, when constructing a fractal portfolio model, in order to correspond to the Markowitz mean-variance model, the investment portfolio $P$ is still composed of two risk assets, $M$ and $N$. The returns are measured by fractal expectation, and risks are measured by fractal variance. Therefore, in the fractal statistical measure, the excess return rates of $M$ and $N$ can be recorded as $R_{M}^{f}=E_{f}\left(r_{M}\right)-E_{f}\left(r_{F}\right)$ and $R_{N}^{f}=E_{f}\left(r_{N}\right)-E_{f}\left(r_{F}\right)$ respectively. The objective function Eq.(7) can be solved according to the operation rules shown in Eq.(3) and Eq.(4), and then the weight of the investment $\omega_{M}^{f}$ and $\omega_{N}^{f}$ shown in the following Eq.(8) are obtained.

$$
\begin{gathered}
\max S_{P}^{f}=\frac{\omega_{M}^{f} R_{M}^{f}+\omega_{N}^{f} R_{N}^{f}}{\left(\omega_{M}^{f}\right)^{2} \operatorname{Var}_{f}\left(r_{M}\right)+\left(\omega_{N}^{f}\right)^{2} \operatorname{Var}_{f}\left(r_{N}\right)} \\
\left\{\begin{array}{l}
\omega_{M}^{f}=\frac{\left[\operatorname{Var}_{f}\left(r_{M}\right)\left(R_{N}^{f}\right)^{2}+\operatorname{Var}_{f}\left(r_{N}\right)\left(2 R_{N}^{f}-R_{M}^{f}\right)^{2}\right]^{0.5}}{\left(R_{M}^{f}-R_{N}^{f}\right)\left[\operatorname{Var}_{f}\left(r_{M}\right)+\operatorname{Var}_{f}\left(r_{N}\right)\right]^{0.5}}+\frac{R_{N}^{f}}{R_{N}^{f}-R_{M}^{f}} \\
\omega_{N}^{f}=\frac{\left(\operatorname{Var}_{f}\left(r_{N}\right)\left(R_{M}^{f}\right)^{2}+\operatorname{Var}_{f}\left(r_{M}\right)\left(2 R_{M}^{f}-R_{N}^{f}\right)^{2}\right)^{0.5}}{\left(R_{N}^{f}-R_{M}^{f}\right)\left[\operatorname{Var}_{f}\left(r_{N}\right)+\operatorname{Var}_{f}\left(r_{M}\right)\right]^{0.5}}+\frac{R_{M}^{f}}{R_{M}^{f}-R_{N}^{f}}
\end{array}\right.
\end{gathered}
$$

Despite the fact the Eq.(8) gives the array weight of each asset under the fractal statistical measure, it is very difficult to directly allocate investment funds by using the array weights, so it is necessary to convert the array weights into numerical weights in order to realize the actual allocation of investment funds, and $\omega_{M}^{f}=\left\langle W_{M}, w_{M}\right\rangle 、 \omega_{N}^{f}=\left\langle W_{N}, w_{N}\right\rangle$ can be written for these. In combination with the previous definition of the fractal statistical measure, the following Eq.(9) holds, that is, $\omega_{M}^{f} \approx W_{M} c^{w_{M}}$ and $\omega_{N}^{f} \approx W_{N} c^{w_{N}}$ hold for larger $c$. At this time, the following Eq. (10) can be used to convert the array weights of risk assets a and b into numerical weights.

$$
\begin{aligned}
& \lim _{c \rightarrow \infty} \omega_{M}^{f} W_{M}^{-1} c^{-w_{M}}=\lim _{c \rightarrow \infty} \omega_{N}^{f} W_{N}^{-1} c^{-w_{N}}=1 \\
& \omega_{i=M, N}^{f}=W_{i} c^{w_{i}}\left(W_{M} c^{w_{M}}+W_{N} c^{w_{N}}\right)
\end{aligned}
$$

At this point, it can be seen that the fractal portfolio model constructed based on the fractal idea not only can theoretically overcome the disadvantages of non-fractal methods, which are inaccurate or even impossible to measure the

\section{DOI: $10.24818 / 18423264 / 55.1 .21 .14$}


returns and risks when the financial market has non-linear characteristics such as non-linearity under the framework of complex financial theory, but also can be applied to actual investment practices by converting the array weights into numerical weights.

\section{Empirical Analysis of the Validity of Fractal portfolio Model}

Based on the reality that the financial system is actually a complex hybrid one and the actual financial market has the characteristics including non-linearity, the fractal portfolio model can relatively better solve the portfolio problem under the fractal statistical measure, and is superior to the traditional Markowitz meanvariance portfolio model. And this paper will further test the effectiveness of the constructed fractal portfolio model through empirical analysis, so as not to depart from the reality. Here we select sample data of six industry indexes of the Shanghai Stock Exchange (i.e., industrial, business, real estate, utility, comprehensive and financial indexes) as risk asset samples, and the national debt index return of China are the risk-free return to investigate the performance of investment portfolios that consist any two of the six indexes. In the selection of the sample interval, in order to include enough complete market conditions, so as to reflect the effect of fractal portfolios in different market conditions and avoid empirical results from deviating from reality, this paper takes data from January 2, 2004 to July 23, 2019 as the entire sample interval, and builds an investment portfolio based on the daily closing price of each year, and observes the return on investment of the constructed portfolio in the next year. In addition, this paper also uses the Markowitz traditional portfolio model as the benchmark portfolio to compare with the performance of the fractal portfolio model analysis. The data comes from the Wind database.

To construct a fractal portfolio, the calculation of the density function of the yield sequence must be done firstly. Then we can calculate the fractal expectations and fractal variance based on previous step, and finally determine the weight of each asset in the fractal portfolio. Based on this, if the density function of the yield sequence $\left\{x_{t}\right\}_{t=1}^{m}$ is $\rho(x)=\rho_{0} x^{-\alpha}, \ln F(x)=\ln \rho_{0}(1-\alpha)^{-1}+(1-\alpha) \ln x$ holds for the distribution function $F(x)$ of the yield sequence. Then we arrange the yield sequence $\left\{x_{t}\right\}_{t=1}^{m}$ from small to large and record it as $\left\{r_{t}\right\}_{t=1}^{m}$, where $p_{t}$ is the cumulative probability corresponding to each yield $r_{t}$. Setting the regression result of $\ln p_{t}$ and $\ln r_{t}$ be $\ln p_{t}=\theta \ln r_{t}+\eta$, if the regression equation has a good fitting $R^{2}$, it indicates that the density function is assumed to be $\rho(x)=\rho_{0} x^{-\alpha}$, and the two parameters $\rho_{0}$ and $\alpha$ of the density function of the yield series can be solved according to $\alpha=1-\theta$ and $\rho_{0}=\theta e^{\eta}$, the density function of the return sequence of 6 types of risk assets in 15 time intervals can be further obtained. Among them, the 
Research on Fractal Portfolio Model under Power-law Distribution of Return Rate

two parameters and the goodness of fit of the density function of the 90 risk asset return series are shown in Table 1 and Table 2 below.

Table 1. Two parameters of the density function for 90 yield series

\begin{tabular}{|c|c|c|c|c|c|c|}
\hline \multirow{2}{*}{ Year } & \multicolumn{2}{|c|}{ Ind } & \multicolumn{2}{|c|}{ Bus } & \multicolumn{2}{|c|}{ Rea } \\
\hline & $\rho_{0}$ & $\alpha$ & $\rho_{0}$ & $\alpha$ & $\rho_{0}$ & $\alpha$ \\
\hline 2004 & 6.524 & 0.368 & 23.814 & 0.132 & 19.657 & 0.142 \\
\hline 2005 & 11.821 & 0.263 & 29.940 & 0.082 & 8.954 & 0.270 \\
\hline 2006 & 14.955 & 0.219 & 14.506 & 0.220 & 8.079 & 0.263 \\
\hline 2007 & 25.179 & 0.032 & 21.139 & 0.072 & 16.399 & 0.051 \\
\hline 2008 & 7.116 & 0.270 & 8.657 & 0.223 & 7.387 & 0.184 \\
\hline 2009 & 28.115 & 0.046 & 19.216 & 0.125 & 5.503 & 0.314 \\
\hline 2010 & 13.896 & 0.232 & 23.385 & 0.109 & 18.938 & 0.151 \\
\hline 2011 & 20.711 & 0.183 & 20.408 & 0.162 & 6.394 & 0.358 \\
\hline 2012 & 20.763 & 0.183 & 15.125 & 0.208 & 8.224 & 0.302 \\
\hline 2013 & 50.139 & 0.050 & 36.826 & 0.046 & 10.276 & 0.244 \\
\hline 2014 & 16.309 & 0.235 & 52.213 & 0.000 & 6.406 & 0.333 \\
\hline 2015 & 25.117 & 0.047 & 26.855 & -0.010 & 6.700 & 0.258 \\
\hline 2016 & 11.200 & 0.296 & 7.732 & 0.323 & 22.644 & 0.126 \\
\hline 2017 & 95.390 & 0.021 & 26.578 & 0.185 & 21.834 & 0.196 \\
\hline 2018 & 17.704 & 0.210 & 29.221 & 0.106 & 24.830 & 0.090 \\
\hline \multirow{2}{*}{ Year } & \multicolumn{2}{|c|}{ Uti } & \multicolumn{2}{|c|}{ Com } & \multicolumn{2}{|l|}{$\mathrm{Fil}$} \\
\hline & $\rho_{0}$ & $\alpha$ & $\rho_{0}$ & $\alpha$ & $\rho_{0}$ & $\alpha$ \\
\hline 2004 & 9.920 & 0.298 & 11.180 & 0.270 & 12.920 & 0.216 \\
\hline 2005 & 20.545 & 0.185 & 11.880 & 0.248 & 10.999 & 0.267 \\
\hline 2006 & 11.150 & 0.265 & 9.087 & 0.274 & 19.284 & 0.100 \\
\hline 2007 & 42.590 & -0.098 & 16.381 & 0.111 & 16.695 & 0.080 \\
\hline 2008 & 7.165 & 0.241 & 10.745 & 0.166 & 9.643 & 0.152 \\
\hline 2009 & 7.997 & 0.296 & 20.494 & 0.100 & 15.025 & 0.133 \\
\hline 2010 & 15.968 & 0.198 & 19.138 & 0.174 & 23.261 & 0.121 \\
\hline 2011 & 29.651 & 0.125 & 10.417 & 0.323 & 17.694 & 0.202 \\
\hline 2012 & 23.174 & 0.179 & 13.103 & 0.275 & 11.585 & 0.266 \\
\hline 2013 & 27.551 & 0.136 & 11.964 & 0.276 & 8.228 & 0.304 \\
\hline 2014 & 22.135 & 0.152 & 10.890 & 0.273 & 7.278 & 0.322 \\
\hline 2015 & 9.742 & 0.206 & 8.219 & 0.269 & 7.345 & 0.274 \\
\hline 2016 & 8.726 & 0.329 & 18.514 & 0.243 & 26.600 & 0.163 \\
\hline
\end{tabular}


Linlin Zhang, Yizhuo Li, Xu Wu, Qianying Feng

\begin{tabular}{lllllll}
2017 & 54.123 & 0.112 & 52.374 & 0.115 & 39.167 & 0.117 \\
2018 & 45.437 & 0.044 & 21.889 & 0.181 & 17.389 & 0.207 \\
\hline
\end{tabular}

Note: The actual value of the business index in 2014 is 0.00024 . Since The data in Table 1 only retains three decimal places, it is displayed as 0 .

Table 2. Goodness of fit of 90 density functions

\begin{tabular}{ccccccccc}
\hline Year & 2004 & 2005 & 2006 & 2007 & 2008 & 2009 & 2010 & 2011 \\
\hline Ind & 0.974 & 0.958 & 0.976 & 0.958 & 0.950 & 0.969 & 0.958 & 0.970 \\
Bus & 0.959 & 0.946 & 0.976 & 0.958 & 0.977 & 0.966 & 0.979 & 0.989 \\
Rea & 0.947 & 0.977 & 0.979 & 0.976 & 0.982 & 0.988 & 0.936 & 0.987 \\
Uti & 0.965 & 0.929 & 0.986 & 0.973 & 0.978 & 0.980 & 0.984 & 0.939 \\
Com & 0.948 & 0.972 & 0.954 & 0.980 & 0.929 & 0.971 & 0.974 & 0.978 \\
Fin & 0.969 & 0.930 & 0.959 & 0.974 & 0.943 & 0.950 & 0.919 & 0.934 \\
\hline Year & 2012 & 2013 & 2014 & 2015 & 2016 & 2017 & 2018 & Mean \\
\hline Ind & 0.959 & 0.929 & 0.959 & 0.929 & 0.959 & 0.957 & 0.976 & 0.959 \\
Bus & 0.975 & 0.970 & 0.962 & 0.941 & 0.980 & 0.976 & 0.937 & 0.966 \\
Rea & 0.971 & 0.966 & 0.955 & 0.933 & 0.926 & 0.959 & 0.945 & 0.962 \\
Uti & 0.948 & 0.941 & 0.945 & 0.935 & 0.977 & 0.964 & 0.902 & 0.956 \\
Com & 0.952 & 0.950 & 0.946 & 0.979 & 0.924 & 0.960 & 0.945 & 0.957 \\
Fin & 0.963 & 0.926 & 0.950 & 0.974 & 0.923 & 0.940 & 0.970 & 0.948 \\
\hline
\end{tabular}

Table 2 has shown that the goodness of fit of the 90 regression equations are all above 0.9 , and the average value is 0.958 , indicating that the density function of the yield series is indeed a power-law form, that is, the two parameters of the density function shown in Table 1 have high reliability. According to Eq. (1) and Eq. (2), combined with the data in Table 1, the fractal expectation and fractal variance of 90 yield series can be calculated. Furthermore, according to Eq. (7) and Eq. (8), the weights of the six types of risk assets when paired in a fractal portfolio model can be obtained in order to get the return rate of the fractal portfolio in the next year. Similarly, according to the traditional expectations and traditional variances of the 90 yields series, using Eq. (6), the weight of each asset under the Markowitz benchmark portfolio can be obtained, and the yield of the benchmark portfolio in the next year is easily obtained. For simplicity and ease of comparison, Table 3 below only lists the difference in returns between the fractal portfolio and the benchmark portfolio. 
Research on Fractal Portfolio Model under Power-law Distribution of Return Rate

Table 3. Yield difference between fractal portfolio and benchmark portfolio

\begin{tabular}{|c|c|c|c|c|c|c|c|c|}
\hline & & & & & & & & \\
\hline & & & & & & & & \\
\hline & & & & & & & & \\
\hline & & & & & & & & \\
\hline & & & & & & & & 076 \\
\hline & 096 & 71 & 63 & .003 & 152 & & 186 & 182 \\
\hline & & & & 9 & & & & \\
\hline & & & & & & & & \\
\hline & & & & & & & & 121 \\
\hline & & & & & & & & \\
\hline & & & & & & & & 0.314 \\
\hline & & & & & & & & \\
\hline & & & & & & & & \\
\hline & & & & & & & & \\
\hline & & & & & & & & 0.258 \\
\hline & & 50 & & & & & & 0.237 \\
\hline Year & 2 & 4 & 2015 & 2016 & 2 & & 2019 & Mean \\
\hline$d_{\mathrm{I}}$ & & & & -2.369 & & & & 200 \\
\hline & & & & & & & & 25 \\
\hline & & & & & & & & \\
\hline & & & & & & & & \\
\hline & & & & & & & & \\
\hline & & 8 & 24 & & 5. & & & 98 \\
\hline & & & & & & & & 74 \\
\hline & & & & & & & & \\
\hline & & & & & & & & \\
\hline & 0 & 2. & S & 6. & 2 & 17 & 25 & .738 \\
\hline & & & & & & & & 51 \\
\hline & & & & & & & & -0.119 \\
\hline & & & & & & & & \\
\hline & & & & & & & & 0.162 \\
\hline & 0 & & , & -2 & 0 & -0 & 0.261 & 0 \\
\hline
\end{tabular}

Note: The actual values of Bus-Rea (2007) and Ind-Uti (2019) are 0.00046 and -0.00018 respectively. Since the data in the table only retains 3 digits after the decimal point, it is displayed as 0 . 
As can be seen from Table 3, among the 225 cases in 15 time intervals, the return rates of the fractal portfolio in 133 cases are higher than those of the benchmark portfolio, accounting for $59.11 \%$ of the total, and is close to $60 \%$. It can be seen that the fractal portfolio model is generally superior and more effective than the benchmark portfolio model. Furthermore, by accumulating the returns of 15 types of portfolios from 2005, we can obtain the cumulative rate of return of the portfolio in 15 time intervals. It can be found that in 173 cases the cumulative rate of return of the fractal portfolio is greater than that of the benchmark portfolio, which amounts to as high as $76.89 \%$ of the total cases. It can be seen that when two portfolio strategies are executed simultaneously in the long term, the superiority of the fractal portfolio over the benchmark portfolio will be more obvious and stable, that is, the fractal portfolio can be used by investors for long-term practices. In order to give a visual aid, the cumulative return rates of 15 portfolio $s$ under the two models are shown in Figure 1. below.

Cumulative Yield Sequence of Risk Portfolios under Two Portfolio Models
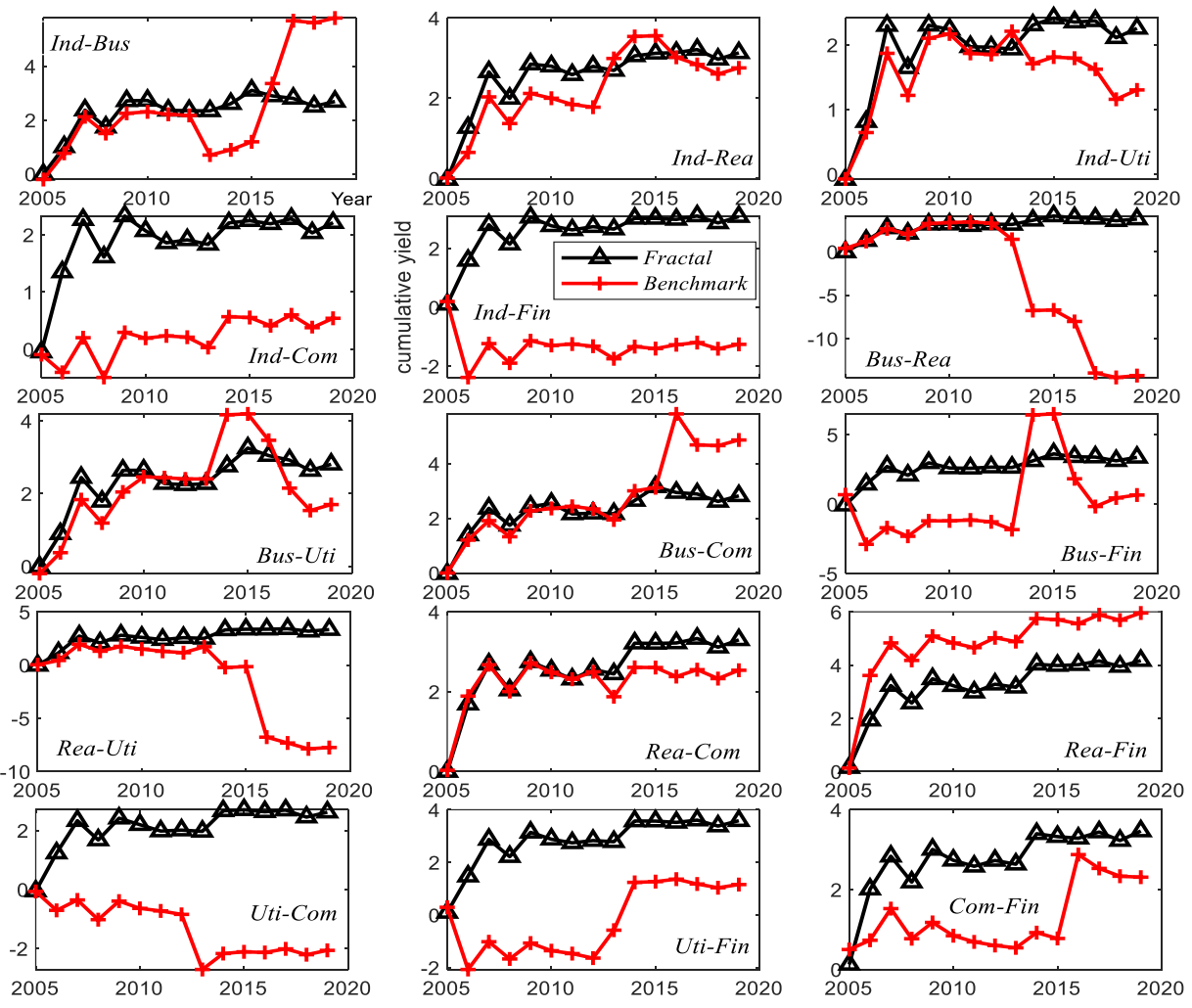

Figure 1. Cumulative Yield Sequence for Fractal Portfolio and Benchmark portfolio 
Research on Fractal Portfolio Model under Power-law Distribution of Return Rate

It can be seen from Figure 1. that in most cases, the cumulative yield of the fractal portfolio is not only higher than that of the cumulative yield of the benchmark portfolio, but also has less fluctuation, which means the cumulative yield of the fractal portfolio is more stable. This shows that compared to the benchmark portfolio model, the fractal portfolio model improves investment performance, and has better robustness, that is, the fractal portfolio model is indeed effective. In order to further verify the effectiveness of the fractal risk asset portfolio, the risk-adjusted performance measurement index, which is, the coefficient of variation is selected as the test object. Table 4 below gives a list of the 15 coefficients of variation of the cumulative return of the risk asset portfolio under the fractal and benchmark portfolio models respectively.

Table 4. Coefficient of variation of cumulative return on fractal risk asset portfolio and benchmark risk asset portfolio

\begin{tabular}{ccccccccc}
\hline \multirow{2}{*}{ Portfolio } & $\begin{array}{c}\text { Ind- } \\
\text { Bus }\end{array}$ & $\begin{array}{c}\text { Ind- } \\
\text { Rea }\end{array}$ & $\begin{array}{c}\text { Ind- } \\
\text { Uti }\end{array}$ & $\begin{array}{c}\text { Ind- } \\
\text { Com }\end{array}$ & $\begin{array}{c}\text { Ind- } \\
\text { Fin }\end{array}$ & $\begin{array}{c}\text { Bus- } \\
\text { Rea }\end{array}$ & $\begin{array}{c}\text { Bus- } \\
\text { Uti }\end{array}$ & $\begin{array}{c}\text { Bus- } \\
\text { Com }\end{array}$ \\
\hline \multirow{2}{*}{ Fractal } & 0.345 & 0.332 & 0.345 & 0.308 & 0.298 & 0.360 & 0.359 & 0.335 \\
Benchmark & 0.758 & 0.439 & 0.385 & 1.505 & -0.390 & -2.365 & 0.546 & 0.550 \\
\hline \multirow{2}{*}{ Portfolio } & Bus- & $\begin{array}{c}\text { Rea- } \\
\text { Fin }\end{array}$ & $\begin{array}{c}\text { Rea- } \\
\text { Uti }\end{array}$ & $\begin{array}{c}\text { Rea- } \\
\text { Com }\end{array}$ & $\begin{array}{c}\text { Eti- } \\
\text { Fom }\end{array}$ & $\begin{array}{c}\text { Uti- } \\
\text { Fin }\end{array}$ & $\begin{array}{c}\text { Com- } \\
\text { Fin }\end{array}$ & \multirow{2}{*}{ Mean } \\
\hline Benchmark & 0.344 & 0.355 & 0.326 & 0.319 & 0.336 & 0.328 & 0.300 & 0.333 \\
\hline
\end{tabular}

As can be seen from Table 4, among the 15 portfolios, in 13 cases the absolute value of the coefficient of variation of the fractal portfolio is smaller than that of the reference portfolio, accounting for $86.67 \%$ of the total. Since the coefficient of variation measures the amount of risk assumed to obtain unit returns, when the positive-negative sign of coefficient of variation coefficients are the same, the smaller the absolute value is, the better the coefficient of variation is; when the sign of variation coefficients are different, the effect of a positive coefficient of variation is better. It also can be seen that under the risk-adjusted performance measurement index, the fractal portfolio model is still better than the benchmark portfolio model, which means that the fractal portfolio model is effective.

To sum up, based on the effectiveness analysis of the fractal portfolio model based on fractal expectation and fractal variance, the fractal portfolio model is superior to the traditional portfolio model and is relatively more stable, whether it is a comparison of investment returns under two portfolio models, a comparison of cumulative returns, or a comparison of coefficient of variation. Therefore, empirical results show that the fractal portfolio model not only has validity in theory, but also is effective based on data analysis, and can be used to improve the performance of investment portfolios.

DOI: 10.24818/18423264/55.1.21.14 


\section{Conclusions}

The main findings of this paper can be summarized as follows. Firstly, based on the fact that the real financial system is a complex hybrid one and there are non-linear characteristics in the real financial market, and the tail of the securities yield rate follows the power-law distribution, the authors construct a portfolio model with fractal expectation and fractal variance as statistical measures of fractal. Secondly, the analytical solution of the model is given and can be used to achieve the purpose of risk diversification and ensuring returns. Thirdly, through empirical analysis, it is found that the fractal expectation and fractal variance can not only overcome the defect that the traditional mean and variance cannot be applied to the tail of power rate distribution, but also is generally better than the mean-variance portfolio model. The fractal portfolio model is effective and more conducive to improve the performance of the investment portfolio.

\section{ACKNOWLEDGEMENTS}

This work is supported by the National Natural Science Foundation of China (71903017), Ministry of Education in China Project of Humanities and Social Sciences (17YJC790168) and Young Core Instructor Training Program of Chengdu University of Technology (KYGG201713).

\section{REFERENCES}

[1] Alexander, G. J., Baptista, A. M. and Yan, S. (2017), Portfolio Selection with Mental Accounts and Estimation Risk. Journal of Empirical Finance, 41(3), 161-186;

[2] Ankargren, S., Bjellerup, Mårten, Shahnazarian, H. (2017), The Importance of the Financial System for the Real Economy. Empirical Economics, 2017, 53(4), 1553-1586;

[3] Black, F. and Litterman, R. B. (1991), Asset Allocation: Combining Investor Views with Market Equilibrium. The Journal of Fixed Income, 1(2), 7-18;

[4] Brandtner, Mario (2013), Conditional Value-at-Risk, Spectral Risk

Measures and (Non-)Diversification in Portfolio Selection Problems -A

Comparison with Mean-Variance Analysis. Journal of Banking \& Finance, 37(12), 5526-5537;

[5] Cont, R. (2001), Empirical Properties of Asset Returns: Stylized Facts and Statistical Issues. Quantitative Finance, 1(2), 223-236;

[6] Goh, J. W., Lim, K. G., Sim, M., et al. (2012), Portfolio Value-at-Risk Optimization for Asymmetrically Distributed Asset Returns. European Journal of Operational Research, 221(2), 397-406; 
Research on Fractal Portfolio Model under Power-law Distribution of Return Rate

[7] Hany, F. (2019), Mean-Variance-Time: An Extension of Markowitz's MeanVariance Portfolio Theory. Journal of Economics and Business, 105888, ISSN 0148-6195, https://doi.org/10.1016/j.jeconbus.2019.105888;

[8] Jung, J. and Kim, S. (2015), An Adaptively Managed Dynamic Portfolio Selection Model Using a Time-Varying Investment Target According to the Market Forecast. Journal of the Operational Research Society, 66(7), 1115-1131; [9] Li, T., Zhang, W. and Xu, W. (2013), Fuzzy Possibilistic Portfolio Selection Model with Var Constraint and Risk-Free Investment. Economic Modelling, 31(Complete), 12-17;

[10] Lim, G., Kim, S. Y., Lee, H., et al. (2007), Multifractal Detrended Fluctuation Analysis of Derivative and Spot Markets. Physica A, 386(1), 259266;

[11] Lux, T., Alfarano, S. (2016), Financial Power Laws: Empirical Evidence, Models and Mechanisms. Chaos, Solitons and Fractals: The interdisciplinary journal of Nonlinear Science, and Nonequilibrium and Complex Phenomena, 88(7), 3-18;

[12] Markowitz, H. M. (1991), Foundations of Portfolio Theory. The Journal of Finance,46(2), 469-477;

[13] Markowitz, H. M. (1952), Portfolio Selection. The Journal of Finance, 7(1), 77-91;

[14] Mandelbrot, B B. (1967), How Long Is the Coast of Britain? Statistical Self-Similarity and Fractional Dimension. Science, 156(3775), 636-638;

[15] Ortobelli, S., Kouaissah, N. and Tomáš, Tichý. (2017), On the Impact of Conditional Expectation Estimators in Portfolio Theory. Computational Management Science, 14(5), 1-23;

[16] Peretz, D. (1971), Thirty-five Years of Change for the Financial System. Futures, 3(4), 0-356;

[17] Samorodnitsky, G. and Taqqu, M. S. (1996), Stable Non-Gaussian

Random Processes: Stochastic Models with Infinite Variance. Journal of the American Statistical Association, 90(430);

[18] Samuelson, P. A. (1967), General Proof that Diversification Pays. Journal of Financial and Quantitative Analysis, 2(1), 1-13;

[19] Scheinkman, J. A. (1994), Nonlinear Dynamics in Economics and Finance. Philosophical Transactions of the Royal Society A: Mathematical, Physical and Engineering Sciences, 346(1679), 235-250;

[20] Wang, Y., Li, L. and Gu, R. (2009), Analysis of Efficiency for Shenzhen Stock Market Based on Multifractal Detrended Fluctuation Analysis.

International Review of Financial Analysis, 18(5), 271-276;

[21] Wang, Z., Huang, X. and Shi, G. (2011), Analysis of Nonlinear Dynamics and Chaos in a Fractional Order Financial System with Time Delay. Computers \& Mathematics with Applications, 62(3), 1531-1539;

DOI: $10.24818 / 18423264 / 55.1 .21 .14$ 
Linlin Zhang, Yizhuo Li, Xu Wu, Qianying Feng

[22] Wu, X., Song, G. H., Deng, Y. and Xu, L. (2015), Study on Conversion between Momentum and Contrarian Based on Fractal Game. Fractals, 23(3), 110 ;

[23] Yuan, Y., Zhuang, X. T. and Jin, X. (2009), Measuring Multifractality of Stock Price Fluctuation Using Multifractal Detrended Fluctuation Analysis. Physica A: Statistical Mechanics and its Applications, 388(11), 2189-2197;

[24] Zhang, Y., Li, X. and Guo, S. (2018), Portfolio Selection Problems with Markowitz's Mean-Variance Framework: A Review Of Literature. Fuzzy Optimization and Decision Making, 17(2), 125-158;

[25] Zhao, P. and Xiao, Q. (2016), Portfolio Selection Problem with Value-atRisk Constraints under Non-Extensive Statistical Mechanics. Journal of Computational and Applied Mathematics, 298, 64-71. 